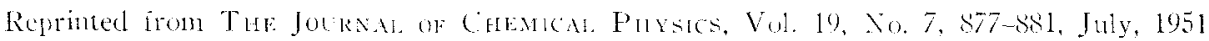

P'rinted in U. S. A.

\title{
Thermodynamics and Chemical Kinetics of One-Dimensional Nonviscous Flow through a Laval Nozzle
}

\author{
S. S. PENNER \\ Guspenhein Iot Propulsion Center, California Institute of Tekhology, Pasadena, Californit
}

(Received February 13, 1951)

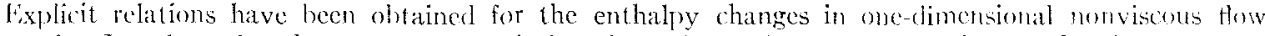
through a Laval nozzle, where arbitrary deviations from thernodynamic equilibrium of chenical composi tion and of internal electronic, vibrational, or rotational energy states may occur. These relations are of interest in connection with calculations on the effect of deviations from equilibrium on performance of jet engines.

Starting with the cquation of continuity for a multicomponent mixture of reacting gases, criteria for nearequilibrium and for near-frozen flow with respect to chemical reactions are derived. The near-equilibrium criteria agree with results obtained previously. The near-frozen flow criteria are new and have not yet been applied to the study of chemical reactions during nozzle flow.
\end{abstract}

\section{INTRODUCTION}

$\mathrm{T}$ HE determination of translational energy for fluid mixtures after expansion through a Laval nozzle involves use of a well-known procedure, at least for the special case of one-dimensional adiabatic expansion with complete chemical equilibrium or without composition change. ${ }^{1-4}$ It has usually been assumed that complete thermodynamic equilibrium is maintained during flow with respect to vibrational and rotational energy states, although numerical calculations for a few representative cases of vibrationally frozen flow have been discussed. ${ }^{5}$ The calculation of exhaust velocity after one-dimensional adiabatic expansion through a Laval nozzle can be carried out for arbitrary distributions of internal energy and for arbitrary composition changes. It is the purpose of Section II to present a systematization of calculations of this type in a convenient form for practical applications.

Closely connected with the essentially thermodynamic calculations of exhaust velocity are the kinetic problems of rates of chemical reactions and rates of adjustment of internal equilibrium states during nozzle flow. Of these kinetic problems only the former has been treated in connection with the evaluation of rocket performance. The methods which have been used fall into two categories: those concerned with exact stepwise numerical calculations ${ }^{2,3,6}$ and those involving the application of near-equilibrium criteria. ${ }^{7-9}$ Exact calculations are extremely laborious and, in view of the lack of adequate information concerning reaction-rate constants, seem hardly worth while. On the other hand, the use of near-equilibrium criteria is at least of qualitative value and in selected cases may even be used for the analysis of a complete propellant system. ${ }^{9} \mathrm{Up}$ to the

${ }^{1}$ F. J. Malina, J. Franklin Inst. 230, 433 (1940).

$2 \mathrm{~S}$. S. Penner and D. Altman, J. Franklin Inst. 245, 421 (1948).

${ }^{3}$ D. Altman and S. S. Penner, J. Chem. Phys. 17, 56 (1949).

1 S. R. Brinkley, Jr, J. Chem. Phys. 15, 107 (1947).

$\checkmark$ S. S. Penner, J. Appl. Phys. 20,445 (1949).

${ }^{6} \mathrm{H}$. Behrens, Z. physik. Chem. 195,1 (1950).

7 S. S. Penner, J. Am. Chem. Soc. 71, 788 (1949).

${ }^{8}$ 5. S. Penner, J. Chem. Phys. 17, 841 (1949).

${ }^{9}$ S. S. Penner, J. Franklin Inst. 249, $441(1950)$ present time only near-equilibrium criteria, that is, methods for allowing rapid screening of reactions which occur so rapidly that they almost maintain concentration changes consistent with local thermodynamic equilibrium, have been used. It is evident that nearfrozen flow criteria should be just as useful as nearequilibrium criteria. Accordingly Section III will be devoted to the development of near-frozen flow criteria, as well as of near-equilibrium criteria, from rather general considerations. With the aid of this new tool for studying kinetic processes during nozzle flow, additional numerical calculations on selected propellant systems should become possible. For complex reactions of the type which occur in the hydrogen-oxygen motor the interdependence of chemical reactions will have to be considered according to a scheme described in an earlier publication. ${ }^{8}$

\section{CALCULATION OF TRANSLATIONAL ENERGY FOR FLUID MIXTURES DURING ADIABATIC FLOW}

The principle of conservation of energy for fluid mixtures, during steady one-dimensional adiabatic flow, may be expressed by the relation ${ }^{10}$

$$
H+\frac{1}{2} u^{2}=\text { constant, }
$$

where $H$ represents the enthalpy per gram of fluid mixture and $u$ is the linear flow velocity. Equation (1) forms the basis for the calculation of exhaust velocitics after flow through a Laval nozzle. In particular, if it is assumed that the translational velocity at the nozzle entrance (conditions at the nozzle entrance are identified by the subscript $c$ corresponding to the chamber temperature $T_{c}$ ) is zero, then the translational velocity at the nozzle exit (identified by the subscript $e$ corresponding to the exhaust temperature $T_{e}$ ) is given by the relation

$$
\frac{1}{2} u_{e}^{2}=H_{c}-I_{c}=\Delta H_{i}{ }^{c} .
$$

It is well known that the numerical value of $u_{e}$ depends on the nature of the flow process and will assume

${ }^{10}$ H. W. Liepmann and A. E. Pucket, Aerodynamics of Compressible Flow (John Wiley and Sons, Inc., New York, 1947). 
different values depending on the extent of chemical reaction and redistribution of internal energy during flow. In order to exhibit this dependence of $u_{e}$ on the flow process in some detail, it is desirable to express $\Delta H_{e}{ }^{c}$ in such a form as to demonstrate explicitely its dependence on the occurrence or nonoccurrence of chemical reactions and internal energy adjustments. The term equilibrium flow will be understood to mean flow in which equilibrium is maintained at each point with respect to chemical reaction, whereas frozen or constant-composition flow means flow without chemical reaction. The state of the electronic, vibrational, and rotational distribution will be identified by a statement concerning the internal energy distribution. Let $H_{0}{ }^{0}$ = enthalpy per gram of fluid mixture if complete chemical and internal equilibrium is maintained for all constituents; $H^{0}=$ enthalpy per gram of fluid mixture if complete chemical equilibrium is maintained for all components but internal-energy lags may exist; $H_{0}=$ enthalpy per gram of fluid mixture if complete internal equilibrium exists for all constituents but the chemical composition may depart from equilibrium. It is convenient to form the following identity

$$
\begin{aligned}
H=H_{0}{ }^{0}-\frac{1}{2}\left[\left(H_{0}^{0}{ }^{0}-H_{0}\right)+\right. & \left(H_{0}-H\right) \\
& \left.+\left(H_{0}{ }^{0}-H^{0}\right)+\left(H^{0}-H\right)\right],
\end{aligned}
$$

whence

$$
\begin{aligned}
\Delta H_{e}{ }^{c}= & \left(H_{0 c}^{0}-H_{0 e}{ }^{0}\right)-\frac{1}{2}\left\{\left[\left(H_{0 c}^{0}-H_{0 c}\right)+\left(H_{0 c}-H_{c}\right)\right.\right. \\
& \left.+\left(H_{0 c}{ }^{0}-H_{c}^{0}\right)+\left(H_{c}^{0}-H_{c}\right)\right]-\left[\left(H_{0 e}{ }^{0}-H_{0 e}\right)\right. \\
& \left.\left.+\left(H_{0 e}-H_{e}\right)+\left(H_{0 e}{ }^{0}-H_{e}\right)+\left(H_{e}^{0}-H_{e}\right)\right]\right\} .
\end{aligned}
$$

Let $\left(H_{0 e}{ }^{0}-H_{0 e}{ }^{0}\right)=\Delta H_{0 e}{ }^{0 c}=$ enthalpy change for complete chemical and internal equilibrium. In general, the fluid mixture of density $\rho$ contains $N$ different chemical species. The equilibrium concentration of the $k^{\prime}$ th species is $C_{k}{ }^{0}$ in moles per cubic centimeter of mixture or $C_{k} \% \rho$ in moles per gram of mixture. The corresponding actual concentrations are $C_{k}$ in moles per cubic centimeter of mixture or $C_{k} / \rho$ in moles per gram of mixture. Setting the molecular weight of the $k^{\prime}$ th component equal to $M_{k}$, it is apparent that

$$
\begin{aligned}
H_{0}{ }^{0} & =\sum_{k=1}^{N}\left(M_{k} C_{k} \% / \rho\right) H_{0 k} \\
H^{0} & =\sum_{k=1}^{N}\left(M_{k} C_{k}{ }^{0} / \rho\right) H_{k} \\
H_{0} & =\sum_{k=1}^{N}\left(M_{k} C_{k} / \rho\right) H_{0}{ }^{k},
\end{aligned}
$$

where $H_{0 k}$ is the enthalpy per gram of the $k$ th component with equilibrium internal energy distribution and $H_{k}$ is the corresponding enthalpy per gram without equilibrium internal-energy distribution. Introducing Eqs. (5), (5a), and (5b) into Eq. (4), the following relation is obtained:

$$
\begin{aligned}
\frac{1}{2} u_{e}^{2}= & \Delta H_{e} e^{c}=\Delta H_{0 e}{ }^{0 c} \\
& -\frac{1}{2} \sum_{k=1}^{N} M_{k}\left\{( 1 / \rho _ { c } ) \left[\left(C_{k c}{ }^{0}-C_{k c}\right)\left(H_{0 k c}+H_{k c}\right)\right.\right. \\
& \left.+\left(C_{k c}{ }^{0}+C_{k c}\right)\left(H_{0 k c}-H_{k c}\right)\right] \\
& -\left(1 / \rho_{e}\right)\left[\left(C_{k e}-C_{k e}\right)\left(H_{0 k e}+H_{k e}\right)\right. \\
& \left.\left.\quad+\left(C_{k e}{ }^{0}+C_{k e}\right)\left(H_{0 k e}-H_{k e}\right)\right]\right\} .
\end{aligned}
$$

Equation (6) is the desired result and can be used directly to calculate $u_{e}$ for the most general type of information which may be available. The utility of Eq. (6) will now be demonstrated by considering a number of special cases, some of which have been employed previously for the calculation of exhaust velocities from conventional rocket motors.

\section{A. Equilibrium Concentration and Equilibrium Internal Energy at $T_{e}$ and $T_{c}$}

In this case $C_{k r}=C_{k c}{ }^{0}, C_{k e}=C_{k e}{ }^{0}, H_{k e}=H_{0 k c}, H_{k e}=I_{0 k e}$. Hence Eq. (6) becomes

$$
\frac{1}{2} u_{e}^{2}=\Delta H_{0 e}{ }^{0 c}=\sum_{k=1}^{N} M_{k}\left[\left(C_{k c}{ }^{0} H_{0 k c} / \rho_{c}\right)-\left(C_{k e}{ }^{0} H_{0 k e} / \rho_{e}\right)\right] .
$$

Equation (7) shows how the exhaust velocity is calculated for equilibrium flow. For practical purposes it may be convenient to replace the enthalpies in $\mathrm{Eq}$. (7) by heat capacity integrals. Thus

$$
\begin{array}{r}
\frac{1}{2} u_{e}^{2}=\sum_{k=1}^{N}\left\{M_{k}\left[\left(C_{k c}{ }^{0} / \rho_{c}\right) \int_{298}^{T_{c}} C_{p k} d T-\left(C_{k e} e^{0} / \rho_{e}\right) \int_{298}^{T_{e}} C_{p k} d T\right]\right. \\
\left.+H_{0 k}{ }^{298}\left[\left(C_{k c}{ }^{0} / \rho_{c}\right)-\left(C_{k_{e}}{ }^{0} / \rho_{e}\right)\right]\right\}
\end{array}
$$

where $C_{p k}$ is the specific heat at constant pressure per gram of $k$ for equilibrium electronic, vibrational, and rotational excitation, and $I_{0 k}{ }^{298}$ denotes the standard heat of formation of species $k$ at $298^{\circ} \mathrm{K}$.

\section{B. Equilibrium Concentration at $T_{c}$ followed by Frozen Flow to $T_{e}$ and Equilibrium Internal Energy at $T_{e}$ and $T_{c}$}

In this case $C_{k c}=C_{k c}{ }^{0}, H_{k c}=H_{0 k c}, H_{k e}=H_{0 k e}, C_{k e} / \rho_{e}=C_{k c}{ }^{0} / \rho_{c}$. Hence $\mathrm{Eq}$. (6) becomes

$$
\frac{1}{2} u_{e}^{2}=\sum_{k=1}^{N}\left(M_{k 0} C_{k c}{ }^{0} / \rho_{c}\right)\left(H_{0 k c}-H_{0 h e}\right) .
$$

Equation (8) is the usual relation for frozen-flow calculations. In terms of heat capacities it becomes

$$
\frac{1}{2} u_{e}^{2}=\sum_{k=1}^{N}\left(M_{k} C_{k c}{ }^{j} / \rho_{c}\right) \int_{T_{e}}^{T_{c}} C_{p k} d T,
$$

since no composition changes occur during flow.

\section{Equilibrium Concentration at $T_{c}$ with Arbitrary Concentration at $T_{e}$ and Equilibrium Internal Energy at $T_{e}$ and $T_{c}$}

In this case $C_{k c}=C_{k c}{ }^{0}, H_{k c}=H_{0 k c}, H_{k e}=H_{0 k e}, C_{k e} \neq C_{k e}{ }^{0}$. Hence Eq. (6) becomes

$$
\begin{array}{r}
\frac{1}{2} u_{e}^{2}=\sum_{k=1}^{N}\left\{M_{k}\left[\left(C_{k r}{ }^{0} / \rho_{c}\right) \int_{298}^{T_{c}} C_{p k} d T-\left(C_{k e} / \rho_{e}\right) \int_{298}^{T_{e}} C_{p k} d T\right]\right. \\
\left.+H_{0 k} 288\left[\left(C_{k c}{ }^{0} / \rho_{c}\right)-\left(C_{k e} / \rho_{\theta}\right)\right]\right\} .
\end{array}
$$




\section{Equilibrium Internal Energy and Arbitrary Concentration at $T_{e}$ and $T_{c}$}

In this case $H_{k c}=H_{0 k c}, H_{k e}=H_{0 k e}, C_{k c} \neq C_{k c}^{0}, C_{k e} \neq C_{k e} e^{0}$. Hence Fi. (6) becomes

$$
\frac{1}{2} u_{e}^{2}=\sum_{k=1}^{N} M_{k}\left[\left(C_{k c} H_{0 k c} / \rho_{c}\right)-\left(C_{k e} H_{v k e} / \rho_{e}\right)\right] .
$$

\section{E. Equilibrium Concentration at $T_{e}$ and $T_{e}$ and Internal-Energy Lag at $T_{e}$}

In this case $C_{k c}=C_{k c}{ }^{0}, C_{k c}=C_{k \varepsilon}{ }^{0}, H_{k c}=H_{0 k c}, H_{k e} \neq H_{0 k e}$. Hence Eq. (6) becomes

$$
\frac{1}{2} u_{e}^{2}=\Delta H_{0 e}{ }^{0 \tau}+\sum_{k=1}^{N}\left(M_{k} C_{k e} e^{0} \rho_{e}\right)\left(H_{0 k e}-H_{k e}\right) .
$$

The term $H_{10 k e}-H_{k c}$ can be conveniently expressed as the sum of electronic, vibrational, and rotational enthalpy lags; i.e.,

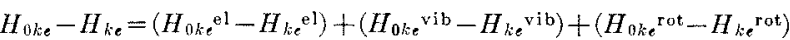

or

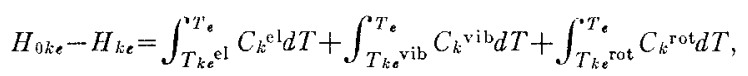

where the superscripts el, vib, and rot denote, respectively, the electronic, vibrational, and rotational contributions to the heat capacity. ${ }^{*}$ The notation $T_{k e}{ }^{\mathrm{ol}}, T_{k e}{ }^{\mathrm{vib}}, T_{k e}{ }^{\mathrm{rot}}$ has been adopted in order to emphasize the fact that, for lagging internal-energy distribution, the electronic, vibrational, and rotational temperatures at the nozzle exit may be different. Since electronic and rotational energy lags are less likely to occur during flow through a Laval nozzle than are vibrational lags, it is of interest to consider Eq. (11) when only vibrational lags occur. In this case

$$
\frac{1}{2} u_{e}^{2}=\Delta H_{0 e} e^{0 c}+\sum_{k=1}^{N}\left(M_{k} C_{k e}{ }^{0} / \rho_{e}\right) \int_{T_{k e}{ }^{\mathrm{vib}}}^{T_{e} C_{k}}{ }^{\mathrm{vib}} d T .
$$

Equation (11a) has been used previously to estimate an upper limit for the effect of vibrational lags on exhaust velocity for equilibrium flow by setting $T_{k e}{ }^{\mathrm{vib}}=T_{c}{ }^{5}$

\section{F. Equilibrium Concentration and Internal Energy at $T_{c}$ Followed by Frozen Flow to $T_{e}$ with Arbitrary Internal-Energy Lags at $T_{e}$}

In this case $C_{k c}=C_{k c}{ }^{0}, C_{k e} / \rho_{e}=C_{h c}{ }^{0} / \rho_{c}, H_{k c}=H_{0 k c}, H_{k e} \neq H_{0 k \text { ke }}$ Hence Eq. (6) becomes

$$
\begin{aligned}
\frac{1}{2} u_{e}{ }^{2}=\Delta H_{v e}{ }^{0 c}+\frac{1}{2} \sum_{k=1}^{N} M_{k}\left\{\left(H_{0 k e}-H_{h e}\right)\left[\left(C_{k e} \% / \rho_{e}\right)+\left(C_{k e}{ }^{0} / \rho_{c}\right)\right]\right. \\
\\
\left.+\left(H_{0 k e}+H_{k e}\right)\left[\left(C_{k e} / \rho_{e}\right)-\left(C_{k c} \% / \rho_{c}\right)\right]\right\} .
\end{aligned}
$$

A comparison of Eqs. (11) and (12) shows very clearly that the effect of vibrational lag on exhaust velocity will depend on the occurrence or nonoccurrence of chemical reaction during flow, a conclusion which was reached previously as the result of numerical calculations. ${ }^{-}$It is possible to derive a quantitative expression for the interdependence of internal and chemical equilibrium. Denoting by $\frac{1}{2} \Delta\left(u_{e}^{2}\right)$ the difference in exhaust velocity between equilibrium and frozen flow for nonequilibrium internal energy at $T_{e}$ and subtracting Eq. (12) from Eq. (11) it is found that

$$
\frac{1}{2} \Delta\left(u_{e}^{2}\right)=\sum_{k=1}^{N} M_{k}\left[\left(C_{k c}{ }^{0} / \rho_{c}\right)-\left(C_{k e}{ }^{0} / \rho_{e}\right)\right]\left[H_{0 k e}-\left(H_{0 k e}-H_{k e}\right)\right] \text {. }
$$

In terms of heat capacity integrals $\mathbf{E q}$. (13) becomes for equilib-

\footnotetext{
* It should be noted that individual electronic, vibrational, and rotational contributions to specific heats are readily calculated by standard statistical methods. See J. E. Mayer and M. E. Mayer, Statistical Mechanics (John Wiley and Sons, Inc., New York,
} 1946). rium electronic and rotational distribution, with the vibrational temperature at the nozzle exit of each of the $k$ components lagging at $T_{c}$

$$
\begin{aligned}
& \frac{1}{2} \Delta\left(u u_{e}^{2}\right)=\sum_{k=1}^{N} M_{k}\left[\left(C_{k \varepsilon} 0 / \rho_{c}\right)-\left(C_{k e} \% / \rho_{\varepsilon}\right)\right] \\
& \times\left[\int_{298}^{T_{\varepsilon}} C_{p k} d T-\int_{T_{c}}^{T_{c}} C_{k}^{\mathrm{vib}} d T+H_{0 k^{298}}\right] .
\end{aligned}
$$

Hence the occurrence of vibrational lag for frozen and equilibrium flow with $T_{k}$ vib $=T_{c}$, has increased the difference between equilibrium and frozen flow by the term

$$
\sum_{k=1}^{N} M_{k}\left[\left(C_{k c}{ }^{0} / \rho_{c}\right)-\left(C_{k e}{ }^{0} / \rho_{e}\right)\right] \int_{T_{s}}^{T_{c}} C_{k}^{\text {vib }} d T .
$$

This last result serves as a good illustration of the utility of Eq. (6) for the analysis of complicated flow problems.

Further results for various specific chemical and internal-energy distributions can be obtained by using the general relation given in Eq. (6) for the solution of particular problems. The more complex the problem at hand, i.e., the more detailed the knowledge concerning concentrations and internal-energy distributions, the more desirable will it be to proceed from Eq. (6) in order to calculate exhaust velocities.

\section{NEAR-EQUILIBRIUM AND NEAR-FROZEN FLOW THROUGH A LAVAL NOZZLE}

As was pointed out in Sec. I, the analysis of kinetic changes during flow has been facilitated in the past by the use of near-equilibrium criteria. Reference to Sec. II shows that full use of available thermodynamic techniques for the calculation of exhaust velocity must await far more complete information concerning the kinetic changes during nozzle flow than are available at the present time. It is therefore of obvious interst to extend the useful kinetic methods by the development of criteria for near-frozen flow. It will be shown in this section how near-frozen as well as near-equilibrium criteria follow from the equation of continuity for a multicomponent mixture of reacting ideal gases.

The equation of continuity for species $k$ of a multicomponent mixture of reacting gases can be written in the form ${ }^{11}$

$$
D C_{k} / D t=\Gamma_{k}+\left(C_{k} / \rho\right)(D \rho / D t)-\nabla \cdot\left[\left(C_{k} \mathbf{V}_{k}\right)\right],
$$

where $D / D t=(\partial / \partial t)+\mathbf{v} \cdot \nabla$ is the euler total time derivative moving with the fluid; $\Gamma_{k}=$ net rate of production of species $k$ in moles per cubic centimeter, as the result of chemical reaction; $t=$ time; $\mathbf{v}=$ mass weighted average velocity $=(1 / \rho) \sum_{k} C_{k} M_{k} \mathbf{v}_{k} ; \mathbf{v}_{k}=$ actual velocity of species $k ; \rho=$ density of fluid mixture $(\mathrm{gm} / \mathrm{cc})$; $C_{k}=$ concentration of species $k(\mathrm{~mol} / \mathrm{cc}) ; M_{k}=$ molecular weight of species $k ; \mathbf{V}_{k}=$ diffusion velocity of species $k$.

For flow through a Laval nozzle the diffusion term is small and may be neglected. Assuming the presence of ideal gases, the equation of state is

$$
\rho=p / \dot{R} T
$$

${ }^{11} \mathrm{C}$. F. Curtiss, and J. O. Hirschfelder, "Kinetic theory of multi-component systems of gases," C. F. 727 (University of Wisconsin, Naval Research Laboratory, Madison, 1947). 
and for adiabatic flow

$$
D p / D T=\gamma p / T(\gamma-1),
$$

whence Eq. (15) becomes

$$
D \ln C_{k} / D t=\Gamma_{k} / C_{k}+[1 / T(\gamma-1)](D T / D \imath) .
$$

Here $p=$ mean hydrostatic pressure; $T=$ temperature; $R^{\prime}=$ gas constant per $\mathrm{g} ; \gamma=$ ratio of specific heat at constant pressure to the specific heat at constant volume, including ${ }^{2}$ any contributions resulting from the occurrence of chemical reactions.

The most general type of chemical reaction may be represented by the relation

$$
\sum_{k} \nu_{k}{ }^{\prime} M_{k}=\sum_{k} \nu_{k}^{\prime \prime} M_{k}
$$

where $\nu_{k}^{\prime}$ and $\nu_{k}^{\prime \prime}$ are the coefficients which multiply the chemical symbol $M_{k}$ of species $k$ in the relation representing the stoichiometry of the reaction. For a reaction of the type shown in Eq. (19) it is apparent that

$$
\Gamma_{k}=\left(\nu_{k}{ }^{\prime \prime}-\nu_{k}{ }^{\prime}\right)\left[k_{f} \prod_{j} C_{j}^{\nu^{\prime}{ }^{\prime}}-k_{b} \prod_{i} C_{j}^{\nu_{j}{ }^{\prime \prime}}\right]
$$

where $k_{f}$ and $k_{b}$ are specific reaction-rate constants for the forward and reverse reactions represented in Eq. (19) by an arrow pointing to the right or to the left, respectively.

From Eqs. (18) and (20) it is apparent that

$$
\begin{aligned}
D \ln C_{k} / D t & =\left(\nu_{k}{ }^{\prime \prime}-\nu_{k}{ }^{\prime}\right) k_{f}\left(\prod_{j} C_{j^{v_{j}}} / C_{k}\right) \\
& \times\left\{1-\left[K_{t}(T) \prod_{j} C_{j^{\nu j^{\prime \prime}}} / \prod_{j} C_{j^{\nu j^{\prime}}}\right]\right\} \\
& +[1 / T(\gamma-1)](D T / D t),
\end{aligned}
$$

where use has been made of the relation

$$
K_{e}(T)=\prod_{j} C_{j}^{n \nu_{j}^{\prime}} / \prod_{j} C_{j}^{n \nu_{j} j^{\prime \prime}}=k_{b} / k_{f}
$$

and the superscript zero indicates that the equilibrium concentrations prevail at the local temperature $T$.

A temperature $T^{\prime}$ is next defined by the relation

$$
K_{e}\left(T^{\prime}\right)=\prod_{j} C_{j^{\nu i^{\prime}}} / \prod_{j} C_{j^{{ }^{\prime} i^{\prime \prime}}}
$$

whence

$$
\begin{array}{r}
D \ln C_{k} / D t=\left(\nu_{k}{ }^{\prime \prime}-\nu_{k}{ }^{\prime}\right) k_{j}\left(\prod_{i} C_{j}{ }^{v^{\prime}} / C_{k}\right) \\
\times\left\{1-\left[K_{e}(T) / K_{e}\left(T^{\prime}\right)\right]\right\} \\
\quad+[1 / T(\gamma-1)](D T / D t) .
\end{array}
$$

Equation (24) represents the desired general result and may now be examined for various special cases.

\section{A. Near-Equilibrium Flow}

Near-equilibrium flow is defined as nozzle flow for which the difference $T^{\prime}-T$ is small during the entire flow process. More specifically it has been defined as flow for which only the first two terms of the Taylor expansion for $K_{e}\left(T^{\prime}\right)$ about $K_{e}(T)$ need be included in calculations. ${ }^{7-9}$ Since $T^{\prime} \geqslant T$, it follows that $K_{\epsilon}\left(T^{\prime}\right)$ $\geqslant K_{e}(T)$ for dissociation reactions and

$K_{e}\left(T^{\prime}\right) \simeq K_{e}(T)+K_{e}(T)\left[d \ln K_{e}(T) / d T\right]_{T}\left(T^{\prime}-T\right)$.

Hence Eq. (24) becomes for near-equilibrium flow, since

$$
\begin{gathered}
{\left[d \ln K_{e}(T) / d T\right]_{T}\left(T^{\prime}-T\right) \ll 1,} \\
D \ln C_{k} / D t=\left(\nu_{k}^{\prime \prime}-\nu_{k}{ }^{\prime}\right) k_{f}\left(\prod_{j} C_{j^{\nu j^{\prime}}} / C_{k}\right) \\
\quad \times\left[d \ln K_{e}(T) / d T\right]_{T}\left(T^{\prime}-T\right) \\
+[1 / T(\gamma-1)](D T / D t) .
\end{gathered}
$$

It has been shown previously that the second term on the right-hand side of Eq. (26) is, in general, negligibly small compared to the first term. ${ }^{6}$ Proceeding in the usual way, ${ }^{6}$ it is readily shown that Eq. (26) leads to the following general criterion for near-equilibrium flow :

$$
\begin{aligned}
T^{\prime}-T=\left[1+\frac{\sum_{j}\left(\nu_{j}^{\prime \prime}-\nu_{j}{ }^{\prime}\right)}{(\gamma-1) T\left(d \ln K_{e} / d T\right) T^{\prime} \simeq T}\right]\left(-\frac{D T}{D t}\right) \\
\times\left[k_{f} \prod_{j} C_{j}^{\nu^{\prime}{ }^{\prime}} \sum_{i}\left(\nu_{j}{ }^{\prime \prime}-\nu_{j}{ }^{\prime}\right)^{2} / C_{j}\right]^{-1} .
\end{aligned}
$$

\section{B. Near-Frozen Flow}

Near-frozen nozzle flow is defined as flow for which $T^{\prime} \sim T_{c}$ where $T_{c}$ represents the temperature at the nozzle entrance. Since $T^{\prime} \leqslant T_{c}$, it follows that $K_{e}\left(T^{\prime}\right)$ $\leqslant K_{e}\left(T_{c}\right)$ for dissociation reactions. More specifically near-frozen flow may be defined as flow for which all terms higher than the second in the Taylor expansion of $K_{e}\left(T^{\prime}\right)$ about $K_{e}\left(T_{c}\right)$ may be neglected. Thus

$$
\begin{array}{r}
K_{e}\left(T^{\prime}\right) \simeq K_{e}\left(T_{c}\right) \\
\quad-K_{e}\left(T_{c}\right)\left[d \ln K_{e}(T) / d T\right] T_{c}\left(T_{c}-T^{\prime}\right)
\end{array}
$$

and Eq. (24) becomes, since

$$
\left[d \ln K_{e}(T) / d T\right] T_{c}\left(T_{c}-T^{\prime}\right) \ll 1,
$$

$D \ln C_{k} / D t=\left(\nu_{k}{ }^{\prime \prime}-\nu_{k}{ }^{\prime}\right) k_{f}\left(\prod_{j} C_{j}^{v^{\prime}{ }^{\prime}} / C_{k}\right)$

$$
\begin{aligned}
& \times\left\{1-\left[K_{e}(T) / K_{e}\left(T_{c}\right)\right]\right. \\
& \left.\times\left[1+\left(d \ln K_{e}(T) / d T\right) T_{c}\left(T_{c}-T^{\prime}\right)\right]\right\} \\
& \quad+[1 / T(\gamma-1)](D T / D t) .
\end{aligned}
$$

Proceeding in the usual way, ${ }^{6}$ the following general 
criterion tor near-frozen flow is obtained:

$$
\begin{aligned}
& {\left[\left(\frac{d \ln K_{e}}{d T}\right)_{T^{\prime} \simeq T_{c}}+\sum_{j}\left(\nu_{j}^{\prime \prime}-\nu_{j}^{\prime}\right) /(\gamma-1) T\right]} \\
& \quad \times\left(-\frac{D T}{D t}\right)=k_{f} \prod_{j} C_{j^{\prime j^{\prime}} \sum_{j}} \frac{\left(\nu_{j}^{\prime \prime}-\nu_{j}^{\prime}\right)^{2}}{C_{j}} \\
& \quad \times\left\{1-\frac{K_{e}(T)}{K_{e}\left(T^{\prime}\right)}\left[1+\left(\frac{d \ln K_{e}}{d T}\right)_{T^{\prime} \simeq T_{c}}\left(T_{c}-T^{\prime}\right)\right]\right\} .
\end{aligned}
$$

\section{Flow with Arbitrary Composition Changes}

Flow with arbitrary composition changes can evidently be described by using a Taylor series expansion of $K_{e}\left(T^{\prime}\right)$ about either $K_{e}(T)$ or $K_{e}\left(T_{c}\right)$ and retaining an adequate number of terms in the power series to assure convergence. Depending upon the particular chemical reaction under study, it may be more convenient to use either a generalized near-equilibrium criterion or a generalized near-frozen-flow criterion. 